\title{
Construyendo situaciones en común a través de tecnologías desobradas
}

\section{Building “in-common” Situations through Inoperative Technologies}

\author{
Wenceslao García PuCHAdes \\ Universidad Politécnica de Valencia \\ wencesgp@gmail.com
}

Recibido: 31/06/2013

Aceptado: 30/05/2014

\section{Resumen}

El siguiente texto supone un acercamiento a las posibilidades que ofrece el desarrollo de la tecnología en el campo de la comunicación para la construcción de comunidades de interacción entre iguales. Con ayuda de la teoría filosófica elaborada por el filósofo francés Jean-Luc Nancy acerca de la "comunidad desobrada", trataremos de mostrar en qué medida una situación de comunicación entre iguales tiene por condición el anonimato de sus miembros, su infinita apertura y su ubicuidad. Partiendo de estas premisas, analizaremos las denominadas redes móviles de comunicación entre pares o P2P (MANET). Nuestra intención es proponer la infraestructura anónima, abierta y ubicua que subyace a estas redes de comunicación como el modelo de un operador necesario para la construcción de situaciones comunicativas basadas en premisas igualitarias.

Palabras clave: Jean-Luc Nancy, comunidad desobrada, redes P2P, comunicación entre iguales, adhocracia.

\begin{abstract}
The following text is an approach to the possibilities offered by the development of technology in the field of communication for the construction of communities of interaction among equals. With the help of the French philosopher Alain Badiou's philosophical theory on the "inoperative community" we intend to show that a situation of communication among equals requires the anonymity of its members, its infinite openness and its ubiquity. Taking these premises as a starting point we shall analyse (USA)/analyse (UK) the so-called mobile networks of communication be-
\end{abstract}


tween peers or P2P (MANET). Our aim is to propose the anonymous, open and ubiquitous infrastructure underlying these communication networks as a necessary operator for the construction of communicative situations based on egalitarian premises.

Keywords: Jean-Luc Nancy, inoperative community, P2P networks, egalitarian communication, adhocracy.

\section{Introducción}

El concepto de "comunidad" o "en-común” está nuevamente de moda. Los avances en las tecnologías aplicadas a la comunicación han favorecido la aparición de nuevas formas de experimentar nuestro "ser-juntos" que nos hacen replantear las discusiones acerca del concepto de "comunidad". Hasta el momento muchas de estas experiencias del "en-común" se fundamentaban en una serie de "lazos comunes" -étnicos, nacionales, ideológicos, sindicales, etc.- que diferenciaban a las diferentes comunidades entre sí1 . Sin embargo, hoy en día, las formas inteligibles que antes parecían garantizarnos un contorno común, estable y duradero han entrado en colapso. La causa de esta crisis reside, en parte, en el carácter dinámico (líquido, diría Baumann) de nuestra sociedad global. En una sociedad como la actual, en la que las tecnologías hacen que nada deje de moverse, tales formas se muestran como lo que son, "puro espectro".

Sin embargo, a pesar de la crisis de estas formas de lo "en-común", todavía nos encontramos con teóricos que no renuncian a pensar el concepto de "comunidad". Tal y como veremos más adelante, muchos de ellos han abordado el concepto de "comunidad" sin acudir a estas categorías transcendentes. Uno de los teóricos contemporáneos que más ha teorizado sobre esta concepción de comunidad desde la inmanencia ha sido el filósofo francés Jean-Luc Nancy a través de su concepto de "comunidad desobrada".

Pero, en nuestra opinión, la teoría de Nancy sobre la comunidad desobrada no sólo resulta interesante como representante del pensamiento de la comunidad desde el plano de la inmanencia que ha caracterizado la filosofía en últimas cinco décadas del siglo $\mathrm{XX}$, sino porque nos ofrece unos criterios que nos permiten identificar la existencia de

\footnotetext{
${ }^{1}$ M. A. Wilson, Technically together: rethinking community within techno-society, Nueva York, Peter Lang Publishing, 2006, pp. 21-23. Para más detalle véase acerca la relación entre la formación de las comunidades y el desarrollo tecnológico véase B. Anderson, Comunidades imaginadas. Reflexiones sobre el origen y la difusión del nacionalismo, traducido por E. L. Suárez, México, Fondo de Cultura Económica, 1991.

${ }^{2}$ P. Pelbart, Filosofía de la deserción: nihilismo, locura y comunidad, Buenos Aires, Tinta Limón, 2009, pp. 21-22; Z. Bauman, Community. Seeking safety in an insecure World, Cambridge, Polity Press, 2001, pp. 1-6.
} 
comunidades comunicativas igualitarias y no autoritarias. Ahora bien, en nuestra opinión, este pensamiento fracasa en la tarea de mostrar cómo es posible construir dichas comunidades de una manera estable y duradera. El problema, veremos, es que la filosofía de la inmanencia nos conduce a una concepción de comunidad igualitaria que relega los criterios para su formación al ámbito de la experiencia subjetiva y privada.Así, en ausencia de criterios objetivos y públicos que permitan legitimarlas, estas comunidades quedan relegadas a ser situaciones acontecimientales y efímeras.

De esta manera, partiendo de lo que a nuestro juicio es el fracaso de la filosofía de la inmanencia para transmitir la posibilidad de crear comunidades igualitarias más allá de situaciones acontecimientales efímeras y sin estructura organizativa, la siguiente ponencia pretende establecer unos criterios que nos permitan pensar en la posibilidad de construir comunidades de comunicación entre iguales de manera consistente y duradera.

El siguiente texto lo estructuraremos en dos bloques principales: en el primero argumentaremos en qué medida es posible extraer de la teoría del filósofo Jean-Luc Nancy sobre la "comunidad desobrada" las condiciones para la construcción de una comunidad comunicativa igualitaria, así como las limitaciones que dicha teoría encierra. En el segundo bloque expondremos en qué medida es posible superar estas limitaciones presentando como paradigma de estas comunidades aquellas que, en los últimos años, se están desarrollando como consecuencia del desarrollo de la tecnología móvil y las redes P2P. Tomando como modelo la infraestructura que constituyen las redes móviles P2P (MANET), pretendemos abandonar la legitimación privada de las comunidades igualitarias, para pensarlas a través de criterios públicos que puedan ser reconocidos por cualquiera de sus miembros.

\section{La comunidad desobrada}

A pesar de la crisis de las categorías transcendentales que antes daban forma a nuestras experiencias en común, algunos filósofos contemporáneos no han renunciado a cuestionarse cual es el significado de "comunidad" y han abordado dicho concepto desde el plano de la inmanencia ${ }^{3}$. En ausencia de categorías abstractas que ofrecieran un significado a la existencia, el pensamiento de la inmanencia considera el ser humano como un ser que, "arrojado" a una cierta situación, es forzado a desarrollar su propio proyecto de existencia con la única ayuda de lo que "tiene-a-

\footnotetext{
${ }^{3}$ Según Lawlor, el pensamiento de la inmanencia tiene origen en las investigaciones realizadas por filósofos como Nietzsche (con su "voluntad de poder"), Bergson (con su "impulso vital"), Heidegger (con su "diferencia ontológica") y, principalmente, Merleau-Ponty (con su revisión de la dialéctica hegeliana y la fenomenología de consciencia husserliana). Cf. L. Lawlor, The Implications of Immanence. Toward a New Concept of Life, New York, Fordham University Press, 2006, pp. 1-3.
} 
mano" . Partiendo de este punto de vista, es posible deducir dos condiciones que son comunes a todo proyecto existencial: 1) su origen contingente, en la medida en que no se puede acudir a entidades transcendentales que justifiquen a priori la razón de su existencia; y 2) su finalidad abierta, porque no puede acudir a ninguna entidad transcendental que le ayude a predecir su fin.

Tomando como base estas premisas, pensadores contemporáneos como Blanchot, Agamben, Virno o Hardt y Negri, han centrado su atención en la esencia co-originaria del ser humano ${ }^{5}$. Sobre las bases del pensamiento heideggeriano, estos teóricos consideran que el ser humano no es ni "el hombre", ni "el sujeto", ni "uno", único y aislado, sino siempre y solamente, el cada uno de los "uno-con-otros".

Pero quizás, haya sido el filósofo francés Jean-Luc Nancy uno de los teóricos que más ha pensado el carácter co-originario de la existencia humana. Para este autor es necesario abordar la existencia del ser humano a partir de su disposición a "ser-conotros", es decir, a "ser-en-común". Para el filósofo francés la existencia humana es "singularmente plural" o "pluralmente singular" porque cualquier ser humano necesita interaccionar con otros para llevar a cabo su proyecto, o usando terminología heideggeriana, para proyectarse en el mundo. Con otras palabras, dado que resulta imposible identificar la existencia de un individuo como un todo de sentido independientemente de los otros, el ser, sólo puede ser pensado como la singularidad de cada uno en relación con los otros ${ }^{7}$.

En muchos de sus trabajos, Nancy caracteriza esta situación "en-común" o comunidad de la existencia desde el paradigma del pensamiento de la inmanencia de acuerdo a tres componentes: el individuo (dasein), los otros y el lugar ${ }^{8}$. Ahora bien, dado que este paradigma renuncia a acudir a categorías transcendentales, esta tarea no puede tener en consideración las propiedades fenomenológicas de estos compo-

\footnotetext{
${ }^{4}$ M. Heidegger, Ser y tiempo, traducción por José Gaos, México D. F., Fondo de Cultura Económica, 1944.

${ }^{5}$ Cf. M. Blanchot, La comunidad inconfesable, traducción por I. Herrera, Madrid, Arena Libros, 2002; G. Agamben, La comunidad que viene, traducción por J. L. Villacañas y C. La Rocca, Valencia, Pretextos, 1996; P. Virno, Gramática de la multitud, traducción por A. Gómez, J. D. Estop, Miguel Santucho, Madrid, Traficantes de sueños, 2003; M. Hardt, y A. Negri, Multitud. Guía y democracia en la era del Imperio, traducción por J. A. Bravo, Buenos Aires, Debate, 2004; y M. Hardt, y A. Negri, Imperio, traducción por A. Bixio, Barcelona, Paidós Ibérica, S.A., 2005.

${ }^{6}$ J.-L. Nancy, Ser singular Plural, traducción por Antonio Tudela, Madrid, Arena Libros, 2006, p. 42.

${ }^{7}$ Ibidem, pp. 44-48.

${ }^{8}$ Cf. J.-L. Nancy, La comunidad desobrada, traducción por P. Perera, Madrid, Arena Libros, 2001; J.-L. Nancy, "The Compearance. From the Existence of 'communism' to the Community of 'Existence"”, en Political Theory, 20(3), agosto, 1992, pp. 371-398; J.-L. Nancy, La comunidad afrontada, en M. Blanchot, La comunidad inconfesable, op . cit., pp. 97-120; y J.-L. Nancy, Ser singular plural, op. cit.
} 
nentes. Desde este punto de vista, esta comunidad de interacción existencial tendrá tres características: 1) es independiente de los rasgos particulares (físicos, intelectuales, habilidades, etc.) de cada uno de sus individuos, ya que cualquiera es capaz de interactuar con cualquier otro con independencia de dichos rasgos; 2) es independiente de los rasgos del colectivo, ya que esta interacción se da con independencia de las propiedades comunes de sus componentes (etnia, ideología, hábitos sociales, etc.); y 3 ) es independiente de las características del sitio donde esta ubicada (nacionalidad, ciudadanía, etc.).

Por tanto, podemos definir la comunidad desobrada como la condición de la existencia humana que posibilita que cualquier individuo pueda proyectarse en el mundo, comunicándose con cualquier otro ser humano sin estar determinado de manera previa por una identidad propia o colectiva, o su pertenencia a un lugar determinado.

Sin embargo, en nuestra opinión, lo realmente interesante del concepto de "comunidad desobrada" es que nos permite establecer las condiciones para pensar en la existencia de una comunidad de interacción entre iguales. La clave de esta igualdad se fundamentaría en el hecho de que, en ausencia de propiedades que los identifiquen como pertenecientes a un individuo, un colectivo o un lugar, sus miembros aparecerían como simples singularidades cualesquiera agrupadas en torno a un proyecto en común. Partiendo de este principio podemos deducir tres rasgos característicos de esta comunidad de comunicación entre iguales. El primero sería el carácter anónimo de sus individuos, en la medida en que éstos carecen de propiedades particulares que los identifiquen. El segundo sería su carácter abierto, en la medida en que carecería de una característica común que nos permita identificarla como un todo cerrado y, por tanto, excluyente. Y el tercero sería su carácter ubicuo, en la medida en que el colectivo se desarrollaría con independencia del lugar. Por tanto, por "comunidad desobrada" podemos entender una categoría filosófica con la que Nancy pretende señalar la igual capacidad del ser humano para poder realizar proyectos en colaboración con otros de una manera anónima, abierta y ubicua.

Ahora bien, para Nancy la "comunidad desobrada" no es una comunidad que limita con otras comunidades sino la condición de ser de toda comunidad. No se trata por tanto de la presentación de una comunidad en torno a un proyecto colectivo determinado sino aquello que posibilita que el proyecto salga a la luz ${ }^{9}$.

En nuestra opinión, esta característica hace inoperativos los principios igualitarios que subyacen a la comunidad desobrada. La razón de esta inoperatividad la debemos buscar en los criterios que legitiman dichos principios. Veámoslo con más detalle.

\footnotetext{
${ }^{9}$ J.-L. Nancy, La comunidad desobrada, op. cit., pp. 135-138.
} 


\section{Limitaciones de la teoría de Nancy acerca de la "comunidad desobrada"}

Tal y como argumentamos anteriormente, el término "comunidad desobrada" nombra la capacidad de "singularidades cualquiera" para "ser-en-común" en torno a un proyecto ubicuo y con fines abiertos. Ahora bien, llevar adelante cualquier proyecto implica una organización colectiva. Una organización que forzaría a que cada uno de sus miembros se identificará con respecto a los otros de acuerdo a su tarea. De manera que, conforme el proyecto evolucionara, los miembros de la comunidad se diferenciarían unos de otros de acuerdo a sus habilidades individuales, a su función y a las facilidades que le ofrecen el lugar en el que viven. Todo ello, aparecería contradictorio con el carácter anónimo, abierto y ubicuo con los que habíamos definido la comunidad desobrada. De manera que, tal y como dice el propio Nancy, la comunidad desobrada solo puede ser concebida como una comunidad que no se puede llevar a cabo, una comunidad de excepción, carente de proyecto, de obra y, por tanto, carente de estructura que fuerce a diferenciar a sus miembros. La comunidad desobrada, afirma el filósofo francés, solo puede ser concebida como la sucesión infinita de interrupciones efímeras y momentáneas ${ }^{10}$.

Se podría afirmar que el principio que subyace a la formación de esta comunidad de la excepción es la afirmación de Bartleby, el escribiente de Melville: "Preferiría no hacerlo" ". Pero, ¿qué quiere decir la afirmación que una y otra vez repetía el escribiente de Melville? "Preferiría no hacerlo" es la expresión de la voluntad de Bartleby de negarse a su rutina laboral diaria: copiar manuscritos y documentos legales en una oficina de abogados. A través de esta frase, Bartleby expresa su voluntad de parar de copiar leyes. Él prefiere no hacerlo y repite con serenidad, una y otra vez, esta fórmula en respuesta a las demandas de su inmediato superior. Para Agamben, el gesto del escribiente representa un acto de desobediencia civil ejemplar: el ejercido por alguien que, a pesar de estar en posesión del poder de hacer algo, decide no ejercerlo $^{12}$. Sin embargo, es importante señalar dos características fundamentales de este gesto: en primer lugar, Bartleby no se niega a copiar la ley en nombre de otra mejor -una ley natural o más justa que merezca ser instituida en lugar de la anterior- sino que simplemente se niega a copiarla; en segundo lugar, el escribiente no lleva a cabo esta resistencia de una manera activa, sino con serenidad: simplemente "prefiere no hacerlo"13.

\footnotetext{
${ }^{10}$ Ibidem, p. 170; J.-L. Nancy, La comunidad afrontada, op. cit., pp. 106-108.

${ }^{11}$ H. Melville, Bartleby, el escribiente, traducido por J. L. Borges, Madrid, Siruela, 1997.

${ }^{12} \mathrm{G}$. Agamben, Homo Sacer. El poder soberano y la nuda vida, traducido por A. Gimeno Cuspinera, Valencia, Pretextos, 1998, p. 67.

${ }^{13}$ J. Whyte, “'I Would Prefer Not to': Giorgio Agamben, Bartley and the Potentiality of the Law", en Law Critique, 20, 2009, pp. 309-324, doi 10.1007/s10978-009-9059-9.
} 
En nuestra opinión la frase de Bartleby es el criterio sobre el que se construye la comunidad desobrada de Nancy: un conjunto de singularidades que, a pesar de ser capaces de identificarse para llevar a cabo un proyecto en común, deciden afirmar "preferiría no hacerlo": preferiría no tener mi propia identidad, preferiría no tener una identidad colectiva y preferiría no pertenecer a ningún lugar concreto. Este gesto de radical desobediencia asegura el carácter genérico de los individuos que la constituyen. Ahora bien, el precio que deben pagar por este gesto es renunciar a una organización estable.

Sin embargo, en nuestra opinión, esta no sería la razón fundamental del aparecer efímero de la comunidad desobrada. Esta razón deberíamos buscarla en la imposibilidad de sus miembros para evaluar los criterios que la legitiman. Tal y como hemos argumentado, el criterio que en última instancia legitimaría esta comunidad se fundamentaría en la necesidad de un consenso de sus miembros en torno al enunciado "preferiría no tener identidad”. Ahora bien, ¿podría pensarse en la durabilidad de una comunidad que tuviera como fundamento este consenso? En nuestra opinión, si somos fieles al modo en que Jean-Luc Nancy piensa este tipo de comunidad, esta tarea sería imposible dado que cada uno de los participantes solo podría verificar su propio compromiso con el enunciado anterior y no el compromiso de los demás. Con otras palabras, en última instancia el criterio que legitima la aparición y persistencia de una comunidad comunicativa igualitaria se fundamentaría en el consenso de voluntad de cada uno de sus participantes de participar en un proyecto compartido sin apelar a su propia identidad, a una identidad colectiva o a la identidad derivada de un lugar. Sin embargo como este consenso no puede ser verificado, este tipo de experiencia en-común sólo puede aparecer para desaparecer.

Nuestra intención, por tanto, es partir de las consideraciones de Jean-Luc Nancy acerca la comunidad desobrada con el objetivo de buscar unas condiciones que saquen del ámbito privado los criterios que fundamentan este tipo de situaciones igualitarias. Se trata, por tanto, de hacerlos públicos y accesibles a cualquiera ubicándolos en el plano material, es decir: el plano de los objetos, de los artefactos, de las infraestructuras y los dispositivos.

En nuestra opinión, los últimos avances tecnológicos aplicados al campo de las comunicaciones móviles, unido al desarrollo de diferentes software informáticos hace esta tarea factible. Tal y como veremos a continuación, las redes móviles de comunicación ad hoc entre pares (en inglés MANET) se presentan como una infraestructura capaz de crear situaciones comunicativas ubicuas que se fundamentan en el anonimato de sus miembros y la participación abierta. En las siguientes líneas argumentaremos en qué medida los proyectos colaborativos que se realizan a través de estas redes nos permiten pensar en la construcción de comunidades de comunicación entre iguales sin que por ello las tengamos que relegar al ámbito del acontecimiento efímero. Para ello mostraremos cómo, en la medida en que dichos proyectos ubican 
los principios que legitiman su carácter genérico en una infraestructura material compartida, no necesitan apelar a enunciados subjetivos imposibles de verificar; lo único que se requiere de sus miembros es la decisión de participar en el proyecto. Veamos en primer lugar en qué consiste esta infraestructura de comunicación que subyacen a las redes móviles ad hoc entre pares.

\section{Redes de comunicación entre pares o peer-to-peer}

Para entender qué es una red móvil de comunicación ad hoc entre pares es necesario entender qué es una red de comunicación entre pares:

"Una arquitectura en red distributiva puede ser llamada una red entre pares (peer-topeer, P-to-P, P2P...), si los participantes comparten una parte de su propios recursos de hardware (es decir, potencia de procesador, capacidad de almacenaje, capacidad de unirse a redes, impresoras...). Estos recursos compartidos se utilizan necesariamente para proveer los servicios y contenido ofrecidos por la red (por ejemplo, compartir archivos o espacios de trabajo para colaborar). Esto servicios son accesibles por otros "pares" directamente, sin necesidad de pasar por entidades intermediarias. Los participantes de tales redes son al mismo tiempo proveedores y solicitantes de recursos (servicios y contenidos)" 14 .

Las redes entre pares o P2P suponen un cambio en relación a la arquitectura estática de los modelos cliente-servidor que caracterizaron los inicios de Internet. Estos modelos descentralizados se han construido como relaciones directas entre nodos a través a una red de distribución masiva. En su versión más pura estas redes han permitido que cada nodo de comunicación tenga la capacidad de ser cliente y servidor. Así, una red $\mathrm{P} 2 \mathrm{P}$ se presenta como un tipo de red comunicativa en la que todos sus nodos tienen el mismo estatus, la misma función y son además libres de asociarse entre ellos. Esto es posible porque en lugar de depender de una estación base que coordina el flujo de información entre cada nodo en la red, cada uno de los nodos individuales envía y recibe estos paquetes de información directamente de los otros nodos ${ }^{15}$.

${ }^{14}$ R. Schollmeier, “A Definition of Peer-to-Peer Networking for the Classification of Peer-to-Peer Architectures and Applications", en Proceedings of the First International Conference on Peer-toPeer Computing (P2Pí01), 2001. En línea, fecha de consulta: 1 de diciembre $2012<\mathrm{http}: / / c s d l . c o m-$ puter.org/comp/proceedings/p2p/2001/1503/00/15030101.pdf >.

${ }^{15}$ Cf. M. Miller, Discovering P2P, San Francisco, Sybex, 2001; D. Barkai, Peer-to-Peer Computing. Technologies for Sharing and Collaborating on the Net, Hillsboro, OR, Intel Press, 2001; K. Aberer, y M. Hauswirth, An overview on peer-to-peer information systems, 2002. En línea, fecha de acceso: 2 de diciembre 2012, <http://lsirpeople. epfl.ch/hauswirth/papers /WDAS2002.pdf>; D. Schoder y K. Fischbach, "Peer-to-peer", en Wirtschaftsinformatik, 44(6), 2002, pp. 587-589; D. Schoder, K. Fis- 
Esta arquitectura descentralizada convierte una red P2P en una malla abierta y autoorganizada que permite la formación espontánea y flexible de redes de comunicación. Se trata de una red abierta porque su topología puede ser potencialmente modificada rápidamente y de manera inesperada por la eliminación o adición de nodos. De esta manera el mal funcionamiento de uno de estos nodos o pares no influye en el correcto funcionamiento de la red, posibilitando su estructura adaptarse a la nueva situación. Por otro lado, proveer un servicio en una red P2P, en la medida en que sus nodos comparten costes en sus recursos compartidos, es más barato, más eficiente y consistente que una red de comunicación cliente-servidor ${ }^{16}$.

De acuerdo a esto, podríamos definir un comunidad P2P como una organización colectiva donde cada uno de sus miembros o pares tiene el mismo rol a la hora de compartir sus recursos. Esta posibilidad de compartir sus recursos es posible gracias a un software específico. Primeramente el software P2P se desarrolló principalmente como aplicaciones que permitían compartir archivos como, por ejemplo, Napster, Gnutella o BitTorrent. Sin embargo, en la últimas décadas han surgido diferentes softwares que aplican el uso de redes distributivas y descentralizadas a diferentes campos: 1) En el intercambio y uso compartido de información a tiempo real (Skype, Ufasoft, Synovel, Wiggio) y de gestión de documentos y sistemas de colaboración (Microsoft's Sharepoint, GroveSite, Organice, Owis, Ubidesk);2) En el aumento y uso compartido de banda ancha para la emisión en streaming (PeerCast, P2P-Radio, SCVI.net); 3) En la gestión compartida de almacenamiento (Oceanstore, TeamWox); 4) En el aumento de la potencia de los buscadores específicos (SETI@home project) ${ }^{17}$.

En los últimos años, el poder disruptivo de la tecnología P2P ha sido utilizado para proporcionar ciertas libertades civiles, como la libertad de expresión, garantizando el anonimato y la privacidad. El proyecto TOR (The Onion Router), por ejemplo, ha desarrollado un software que ayuda a garantizar el anonimato de sus usuarios a través de una red P2P ocultando su localización de su número de identificación en red o IP a través de un sistema de enrutación por capas. Otros proyectos similares como Publius, RedRover y FreeHaven permiten la creación de redes a través de las cuales el contenido puede ser publicado de manera anónima ofreciendo resistencia contra los intentos de adversarios poderosos de encontrar y destruir los datos almacenados. Estos

chbach y Ch. Schmitt, "Core Concepts in Peer-to-Peer Networking”, en R. Subramanian y B. Goodman (eds.), P2P Computing: The Evolution of a Disruptive Technology, Hershey, Idea Group Inc., 2005, pp. 1-26; R. Schollmeier, op. cit.

${ }^{16}$ Cf. X. Shen, H. Yu, J. Buford y M. Akon, Handbook of Peer-to-Peer Networking ( $1^{\text {a }}$ ed.) Nueva York, Springer, 2009; H. M. N. D. Bandara y A. P. Jayasumana, "Collaborative Applications over Peer-to-Peer Systems-Challenges and Solutions", en Peer-to-Peer Networking and Applications, 6(3), septiembre 2013, pp. 257-276; R. Schollmeier, op. cit.; D. Schoder, K. Fischbach y Ch. Schmitt, op. cit.

${ }^{17}$ D. Schoder, K. Fischbach y Ch. Schmitt, op. cit., pp. 7-15. 
ejemplos de software P2P son muy adecuados para la formación de colectivos disidentes en regímenes totalitarios, en la medida en que les permiten informar contra el discurso oficial sin miedo a represalias. Sin embargo, tal y como se ha mostrado en los acontecimientos de Oriente Medio y algunos países asiáticos, estas redes son inútiles si funcionan haciendo uso de infraestructuras de comunicación preexistentes, que al estar controladas por el gobierno, pueden ser monitorizadas y por tanto bloqueadas ${ }^{18}$.

\section{Redes móviles ad hoc de comunicación entre pares}

Para hacer frente a estos problemas de censura han surgido en los últimos años algunos proyectos que aplican los últimos avances tecnológicos en telefonía móvil y conexiones inalámbricas a la creación de redes autónomas de comunicación P2P. Un ejemplo de estos proyectos es Commotion Wireless. Commotion es un software de código abierto que utiliza dispositivos cotidianos como teléfonos móviles, ordenadores portátiles y routers inalámbricos para crear una red de comunicación distributiva o P2P. El principal objetivo de este proyecto es conseguir desarrollar una red peer-topeer con independencia de las infraestructuras comunicativas convencionales de manera que no pueda ser monitoreada y controlada por los regímenes opresivos ${ }^{19}$.
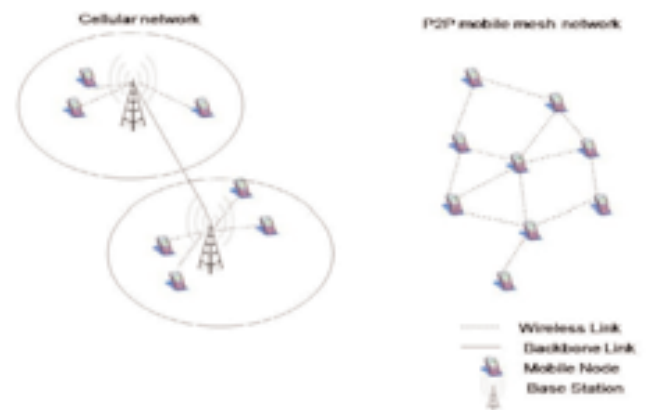

Figura 1: Red de telefonía móvil basada en una arquitectura tradicional contra una red de telefonía móvil P2P20.

\footnotetext{
${ }^{18}$ A. Perreau, "Commotion: A P2P alternative to ISPs", en Eidrien on Technology, 2011. En línea, fecha de acceso: 8 de marzo del 2013, <http://eidrienontech.blogspot.com.es/2011/09/commotionp2p-alternative-to-isps.html>; R. King, "Building a Subversive Grassroots Network", en IEEE Spectrum. Inside Technology, 2011. En línea, fecha de acceso: 7 de marzo del 2013, <http:// spectrum. ieee.org/telecom/internet/building-a-subversive-grassroots-network $>$.

${ }^{19}$ R. King, op . cit.

${ }^{20}$ K. Ranganathan y A. Sarin, “A Voice for the Voiceless: Peer-to-peer Mobile Phone Networks for a Community Radio Service”, en Information Development, 28(1), 2012, pp. 68-79, aquí p. 69, doi: $10.1177 / 0266666911424073$.
} 
El tipo de red que subyace en el proyecto Commotion Wireless ha sido llamado en el ámbito de las telecomunicaciones "redes móviles ad hoc" (MANET) ${ }^{21}$. Sin embargo, tal y como nos sugieren algunos teóricos, este tipo de redes no es nuevo ${ }^{22}$. A comienzos de los setenta, algunos proyectos ya hicieron uso de redes inalámbricas ad hoc en entornos donde no existía una infraestructura que posibilitara la comunicación (por ejemplo, escenarios bélicos o lugares afectados por desastres). La característica principal de estas redes era su movilidad y ubicuidad. El término "ad hoc" señalaba precisamente que este tipo de redes se establecían para un servicio especial y, en ocasiones, efímero (por ejemplo, enviar un video en tiempo real en el campo de batalla o establecer una videoconferencia en lugares de rescate). Además, dada la complejidad de los escenarios, los protocolos utilizados por este tipo de redes ad hoc debían permitir autoconfigurarse para poder ajustarse a los cambios requeridos por el entorno donde se desarrollaba su misión. Se trataban, por tanto, de infraestructuras ubicuas, flexibles y descentralizadas cuya existencia dependía de la duración de la misión para la que habían sido creadas.

Con el desarrollo de la aplicación de la tecnología inalámbrica a dispositivos móviles -teléfonos móviles, PDA, etc.-, las redes MANET expandieron su aplicabilidad y alcance a escenarios comerciales y a asuntos cotidianos ${ }^{23}$. En la década de los setenta el rápido avance de la tecnología de la radio posibilitó el desarrollo de un sistema de comunicaciones inalámbrico que permitió cubrir las necesidades de algunos profesionales para permanecer comunicados mientras se desplazaban. Posteriormente, el desarrollo de la tecnología inalámbrica local (principalmente, IEEE 802.11) y servicios de intercambio de datos para telefonía móvil (GPRS, 1xRTT y UMTS) permitieron hacer un uso cuotidiano de Internet (por ejemplo, conferencias, $e$-mail, e-commercing, $e$-bank, etc.) desde cualquier terminal con independencia del lugar donde esta se encontrara. La tercera fase en esta evolución de las redes inalámbricas permite el acceso a Internet a través de una red de comunicación distributiva o P2P en áreas donde no existe una infraestructura preexistente o donde ésta no cubre las necesidades de la comunidad porque ha dejado de funcionar o porque se encuentra controlada por organismos que impiden su libre utilización. Para ello, solo uno de los

\footnotetext{
${ }^{21}$ Cf. P. Mohapatra y S. Krishnamurthy (ed.), Ad Hoc Networks: Technologies and Protocols, Boston, Springer Science + Business Media, Inc., 2005; R. Sharma, H. Minakshi y K. Gupta, "Mobile Ad Hoc Networks - A Holistic Overview", en International Journal of Computer Applications, 52(21), 2012, pp. 31-36; K. Ranganathan y A. Sarin, op. cit.

${ }^{22}$ M. Gerla, "Ad Hoc Networks", en P. Mohapatra y S. Krishnamurthy (eds.), Ad Hoc Networks: Technologies and Protocols, Boston, Springer Science + Business Media, Inc., 2005, pp. 1-22, aquí p. 2.

${ }^{23}$ Cf. M. Gerla, op . cit., pp. 3-4; T. Back, "P2P Information Sharing in Mobile Ad-hoc Networks", en Seminar on Internetworking 2004, 2005. En línea, fecha de consulta: 23 de febrero de 2013, $<$ http://www.tml.tkk.fi/Publications/C/18/back.pdf>.
} 
nodos que forman parte de dicha red se debe conectar a Internet - por ejemplo a través de router con una antena direccional que alcance un rango de decenas de kilómetros- y compartir la señal con todos sus miembros. Esto es posible gracias a un software P2P que permite que cada nodo actúe como router de manera que pueda compartir los datos con otros nodos que no tengan acceso directo a Internet.

Hoy en día, el uso del software P2P en las redes móviles ad hoc de última generación puede ser aplicado a cualquier ámbito que se quiera beneficiar de la posibilidad de compartir recursos e información en tiempo real de una manera autónoma, flexible y ubicua. Alguna de estas aplicaciones se está dando en la construcción logística de escenarios bélicos ${ }^{24}$, la resolución de situaciones de emergencias y rescate ${ }^{25}$, el intercambio de información y recursos multimedia gratuito ${ }^{26}$ y el activismo democrático ${ }^{27}$.

Desde nuestro punto de vista, y retomando conceptos abordados anteriormente, todos estos proyectos se caracterizan por sostenerse sobre una infraestructura que ofrece la posibilidad crear comunidades comunicativas anónimas, abiertas y ubicuas: se trataría de comunidades anónimas, en la medida en que, tal y como nos ha mostrado el sistema de enrutación desarrollado por el proyecto TOR y retomado por el proyecto Commotion Wireless, cada uno de estos nodos podría hacer uso de la red ocultando su identidad; es una comunidad abierta, en la medida en que, tal y como ocurre en cualquier red distributiva $\mathrm{P} 2 \mathrm{P}$, su adaptabilidad permite que cualquier usuario pueda unirse a ella sin que por ello se altere su eficiencia; y es una comunidad ubicua, en la medida en que posibilita una interacción entre diferentes terminales con independencia de las infraestructuras de comunicación fijas establecidas. Finalmente, estas características, unidas al hecho de que su organización descentralizada les confiere a cada uno de sus nodos la misma función y el mismo estatus dentro de la red, las convierten en comunidades de comunicación entre iguales. En resumen, las redes móviles ad hoc P2P, al igual que la comunidad desobrada, teorizada por Nancy, aparecería como la condición anónima, abierta y ubicua, para que cualquier ser humano desarrolle un proyecto colaborativo siguiendo unos principios igualitarios. Pero veamos con más detalle en qué medida este resultado contribuye a la tarea que nos proponíamos al comienzo del artículo.

\footnotetext{
${ }^{24}$ M. Gerla, op. cit., pp. 8-9; Cf. T. Back, op. cit., y L. Williams y A. Kupetz, "The 4G soldier new developments in military mobile communications", en Mobile Dev\&Desing, junio, 2003. En línea, fecha de acceso: 2 de diciembre de 2012, <http://mobiledevdesign.com/hardware_news /radio_soldier_new_developments/>.

${ }^{25}<$ http://www.virve.com/>

${ }^{26}$ M. Kotadia, "Researchers developing free mobile mesh network", en itnews for Australian business, octubre, 2009. En línea, fecha de consulta: 23 de marzo de 2013, <http://www.itnews.com.au /News/157220,researchers-developing-free-mobile-mesh-network.aspx $>$.

${ }^{27}<$ https://commotionwireless.net/>; Cf. A. Perreau, "Commotion: A P2P alternative to ISPs", op. cit.
} 


\section{Conclusión}

Anteriormente habíamos argumentado cómo la teoría de Nancy acerca de la comunidad desobrada nos permitía extraer los criterios que legitiman las comunidades comunicativas entre iguales. Este tipo de comunidad dependía de que sus elementos se presentaran como carentes de identidad individual, colectiva o situacional. Sin embargo, su enfoque existencialista acababa relegando la legitimación de dichos criterios al ámbito subjetivo y, por tanto, privado, impidiendo así que pudieran ser verificados. Todo ello limitaba la existencia de tales comunidades igualitarias a ser meros acontecimientos efímeros. Así, habíamos hipotetizado que para poder pensar en la existencia de situaciones comunicativas igualitarias estables y duraderas sería necesario hacer públicos los criterios que las legitiman de manera que puedan ser corroboradas por cualquiera. Se trataba de buscar una infraestructura comunicativa que tuviera estas características sin necesidad de apelar a la voluntad de los individuos.

A lo largo de estos últimos párrafos hemos tratado de argumentar que la infraestructura que subyace en las redes móviles de comunicación por pares respondía a estas características. Estas redes se muestran como la condición de una comunicación anónima, abierta y ubicua gracias a un dispositivo formado por varios componentes materiales: una infraestructura (fija o móvil) que permite crear las condiciones físicas para la comunicación en red (es decir, dispositivos móviles, portátiles, PDAs, routers, cableado); un sistema de comunicación e información que permita la interacción descentralizada entre los diferentes agentes cooperantes (es decir, tecnología inalámbrica local y protocolos de intercambio de datos); un software que posibilita la cooperación autónoma entre los usuarios sin intermediarios (es decir, uso de software P2P en wikis, blogs o redes sociales); y una infraestructura legal que evita la apropiación privada de sus recursos (por ejemplo, The General Public License (GPL), que prohíbe la apropiación del código del software, la iniciativa de código abierto o ciertas versiones de Creative Commons License) ${ }^{28}$.

En nuestra opinión esta comunidad de objetos nos ofrecen una base consistente para alejar las situaciones de comunicación igualitarias del ámbito de la excepcionalidad efímera en la medida en que nos permite presentar públicamente los criterios que la legitiman. Con otras palabras, la infraestructura que constituye las redes móviles P2P -es decir, terminales móviles, tecnología inalámbrica, software y leyes- materializa aquello que Nancy ha llamado "comunidad desobrada" y que nosotros hemos señalado como la condición de colaboración entre singularidades cualesquiera con independencia de su identidad individual o colectiva y del lugar al que pertenezcan.

\footnotetext{
${ }^{28}$ M. Bauwens, "The Political Economy of Peer Production", en A. Kroker y M. Kroker (eds.), 1000 Days of Theory, 2005. En línea, fecha de acceso: 2 de diciembre de 2012, <http://www.ctheory.net/articles .aspx $? \mathrm{id}=499>$.
} 
Podríamos decir que la infraestructura que subyace a este tipo de redes es una infraestructura comunicativa desobrada que posibilita que cualquier individuo pueda llevar a cabo un proyecto colaborativo -artístico, científico, político o empresarialsiguiendo unas premisas igualitarias. Ahora bien, a diferencia de lo expuesto en la teoría de Nancy, los criterios que legitiman su carácter igualitario no dependerán de la verificación de enunciados privados ("preferiría no hacerlo"), sino de la decisión de los diferentes usuarios de incorporarse a ellos. Y esto es así, porque su carácter anónimo, abierto y ubicuo no depende de los sujetos sino que se encuentra encarnado en los propios dispositivos que posibilitan esta comunicación. De manera que cualquiera que participe en proyectos colectivos a través de estas infraestructuras ya está aceptando los criterios que legitiman una comunicación igualitaria.

Ahora bien, cada proyecto colaborativo de este tipo evolucionará de acuerdo a sus objetivos organizándose de una manera determinada. De esta manera sus miembros se irán identificando con unos fines colectivos y con unas tareas particulares de acuerdo a sus propias habilidades y conocimientos y al contexto en el que viven. Sin embargo esto no contradice el carácter anónimo, abierto y ubicuo de la infraestructura comunicativa, que permanece siendo desobrada y, por tanto, desligada de cualquier lugar o identidad particular y colectiva.

En nuestra opinión, esta síntesis entre una infraestructura sin identidad, igualitaria y carente de proyecto y una estructura identificada, jerárquica y ligada a un proyecto específico, da lugar a un tipo de organización similar a lo que hace algunas décadas se llamó "adhocracia"29. En 1970 Toffler utilizó este término para referirse a la colaboración flexible entre expertos de diferentes ámbitos que se producía para llevar a cabo un proyecto. Esta colaboración se caracterizaba por poseer una organización jerárquica que estaba ligada al objetivo de dicho proyecto, de manera que cuando éste acaba, su jerarquía también desaparecía ${ }^{30}$. Veinte años después Jonathan Grudin reintrodujo el término "adhocracia" para identificar a aquellas organizaciones de profesionales descentralizadas que se desplegaban en pequeños equipos en respuesta a condiciones cambiantes y ambientes dinámicos y complejos ${ }^{31}$. En el pasado, dicho término iba ligado a industrias creativas, como la publicidad, sin embargo, en los últimos años, algunos teóricos comienzan a asociar el término "adhocracia" con el modo en que diferentes colectivos se organizan para realizar sus proyectos a través de Internet ${ }^{32}$.

\footnotetext{
${ }^{29}$ R. Waterman, Adhocracy: The Power to Change, Nueva York y Londres, W Norton \& Company Inc., 1992.

${ }^{30}$ A. Toffler, Future Shock, Nueva York, Bantam Books, 1970, pp. 124-151

${ }^{31}$ J. Grudin, "Groupware and Cooperative Work: Problems and Prospects", en Brenda Laurel (ed.), The Art of Human-Computer Interface Design, Boston, Addison-Wesley, 1990, p. 183.

${ }^{32}$ D. Ince, "Adhocracy", A Dictionary of the Internet, 2001. En línea, fecha de acceso: 30 de noviembre de 2012, <http://www.encyclopedia.com/doc/1O12-adhocracy.html>.
} 
En nuestra opinión, los proyectos colaborativos realizados con redes móviles P2P representan el paradigma de este modelo de organización. Estos proyectos presentan una síntesis de dos sistemas de organización opuestos: el horizontal (o igualitario) y el vertical (o jerárquico). El sistema de organización horizontal se encuentra presente en la arquitectura de su infraestructura, mientras que el sistema vertical se da en la distribución de roles y de jerarquías que se realiza a la hora de desarrollar un proyecto. El resultado de esta síntesis disyuntiva es un modelo organizativo ad hoc que no sólo es efectivo para resolver problemas específicos en contextos cambiantes, sino que, además, evita la consolidación de liderazgos y estructuras jerárquicas.

Para acabar, nos gustaría señalar la importancia que tiene las investigaciones que se están realizando en el ámbito de la tecnología y de las ciencias informáticas aplicadas a las comunicaciones para la teoría política, pues de ellas se derivan nuevos modelos de organización social que tratan de actualizar aquellos que en el pasado fracasaron. La historia de la humanidad está llena de diferentes proyectos colectivos que han tratado de experimentar formas de organización igualitarias. Sin embargo muchos de estos proyectos han fracasado desapareciendo o dando lugar a sistemas de organización nuevamente desigualitarios y autoritarios ${ }^{33}$.

¿Cómo entender estos fracasos? Quizás podríamos entender estos fracasos partiendo de la hipótesis argumentada en esta ponencia; quizás todas estas experiencias han fracasado a la hora de hacer público el criterio que permitiera legitimar una situación comunicativa entre iguales; y, quizás, esto haya sido así porque en el pasado no existía la infraestructura comunicativa adecuada para ello. Con los últimos avances en el ámbito de las redes de comunicación y tecnologías móviles esta tarea parece posible. Este tipo de redes, todavía en fase experimentación, nos presentan la idea de que cualquier individuo que haga uso de ellas se convierte en una singularidad cualquiera. Este es el punto de origen que comparten todos los miembros que deciden participar en un proyecto con esta infraestructura comunicativa. La voluntad de un individuo de participar en una situación comunicativa igualitaria queda verificada en el momento en que dicho individuo hace uso de esa estructura. Tomando este punto como su origen genérico, ubicuo y abierto, cualquier individuo podrá organizarse junto con otros con la intención de llevar a cabo un proyecto colectivo atendiendo a sus virtudes, habilidades y conocimientos. Todo ello con la característica de que la identidad o estatus que ha conseguido en la colaboración de dicho proyecto desaparecerá en el momento en que participe otro proyecto colaborativo $\mathrm{P} 2 \mathrm{P}$; volviendo, nuevamente, al punto en el cualquier singularidad es igual a cualquier otra.

Esta es en nuestra opinión una de las principales innovaciones que estos modelos organizativos introducirían en la teoría política. Ante la alternativa de elegir entre

\footnotetext{
${ }^{33}$ A. Badiou, Circonstances 5. L'hypothèse communiste, París, Nouvelles Éditions Lignes, 2009.
} 
modelos igualitarios carentes de estructuras jerárquicas y modelos autoritarios con estructuras jerárquicas estables, proyectos como Commotion Wireless proponen una infraestructura comunicativa igualitaria estable que posibilita una estructura organizativa jerárquica, ad hoc y flexible. Esta estructura quedaría legitimada no tanto por la identidad de sus participantes, del colectivo o del lugar en el que se originan, sino por el conocimiento, las habilidades y los recursos que estos demuestran durante la duración del proyecto. Y aunque es cierto que el uso de esta tecnología está todavía en fase de experimentación, y todavía deben mejorar en el campo de la accesibilidad, seguridad y verificabilidad, muchos de estos proyectos albergan nuevas esperanzas en el campo de los estudios políticos-e-democracy,e-citicenship o e-politics - y en el desarrollo de nuevas vías más directas de participación ciudadana ${ }^{34}$.

\footnotetext{
${ }^{34}$ Cf. A. Calderano, "Empirical Analysis of Political Spaces on the Internet: The Role of E- Mailing Lists in the Organization of Alter-Globalization Movements", en International Journal of EPolitics (IJEP), 1(4), 2010, pp. 73-87, doi: 10.4018/jep.2010102205; P. Collin, "Building and Connecting to Online Communities for Action: Young People, ICT and Everyday Politics", en International Journal of E-Politics (IJEP), 1(3), 2010, pp. 1-18, doi:10.4018/jep.2010070101; C. S. Leberknight, M. Chiang y F. M. Wong, "A Taxonomy of Censors and Anti-Censors Part II: AntiCensorship Technologies”, en International Journal of E-Politics (IJEP), 3(4), 2012, pp. 20-35, doi:10.4018/jep.2012100102.
} 